\title{
Ultrastructure of a Clinical Isolate of Staphylococcus Aureus Small Colony Variant and its Revertant
}

\author{
H. Adler, ${ }^{*}$ E. M. Schraner, ${ }^{* *}$ R. Frei, ${ }^{*}$ and P. Wild ${ }^{* *}$ \\ * Microbiology Laboratory, University of Basel Hospitals and Clinics, Petersgraben 4, 4031 Basel, \\ Switzerland \\ ** Electron Microscopy, Institutes of Veterinary Anatomy and of Virology, University of Zürich, \\ Winterthurerstrasse 260, 8057 Zurich, Switzerland
}

Small colony variants of Staphylococcus aureus (SA-SCVs) isolated from various clinical specimens are linked to persistent and recurrent infections. SA-SCVs are characterized by slow growth, small colonies, and by decreased pigmentation and hemolytic activity. The isolate we describe here was recovered from the skin ulcer of a diabetic patient. In contrast to various other reports auxotrophy of the SA-SCV for hemin, menadion or thymidin was not observed. However growth was enhanced by dispersing a thin layer of Tween 80 on the agar surface. Subculturing of SA-SCVs revealed that a varying proportion reverted to the normal colony form.

Electron microscopy of bacteria arrested by metal mirror freezing of colonies in situ or of bacterial suspensions followed by freeze substitution [1] showed that cells of normal colonies were spherelike cells comprising a thin wall and a cytoplasm with areas of regular rough granular appearance interspersed with small areas with fine granular appearance. Many of the cells were in mitosis (Figs. 1A, 2A). Colonies of SA-SCVs comprised mainly thick-walled cells with dense granular appearance at the periphery, and fine granular or fibrillar architecture at the center (Figs. 1B, 2BC). Occasionally the fibrils were concentrically arranged. In addition there were smaller cells consisting of a dense cytoplasm, and a thick cell wall. Many of the cells were in early to late mitosis. The splitting system was indistinct (Fig. 2AB) compared to that in control cells (Fig. 2A). Furthermore, alterations were found in the splitting process per se, and the shape and architecture of cells late in mitosis. Cells with dense cytoplasm and irregular shape are assumed to be dying cells. In samples harvested in situ colonies of SA-SCVs contained many cell ghosts.

The electron microscopic data indicate that cytoplasmic architecture and mitotic division are disturbed in thick-walled cells of the SA-SCV. Division seems to get stuck, and cells disintegrate resulting in cell ghosts as demonstrated in situ. Furthermore, small cells with more compact cytoplasm occurred in the small colonies much more frequently than in the normal colonies. Complete division in these cells is probably slower since, compared with cells of normal colonies, many intermediate stages of division were found. Accordingly, separation of the daughter cells also seems to take longer. Cells may even disintegrate during separation. Enlargement of cells and abnormal forms among dividing cells of a SA-SCV was described earlier [2]. Incomplete and multiple cross walls were found in a thymidin dependent SA-SCV [3]. We describe here for the first time cell wall thickening in a SA-SCV and show details in failure of mitosis.

\section{References}

[1] P. Wild et al., Microsc. Res. Techn. 39 (1997) 297

[2] R. J. Bulger and R. E.Bulger, J. Infect. Dis. 125 (1972) 1244

[3] B. C. Kahl et.al., J. Clin Microbiol. 41(2003) 410 

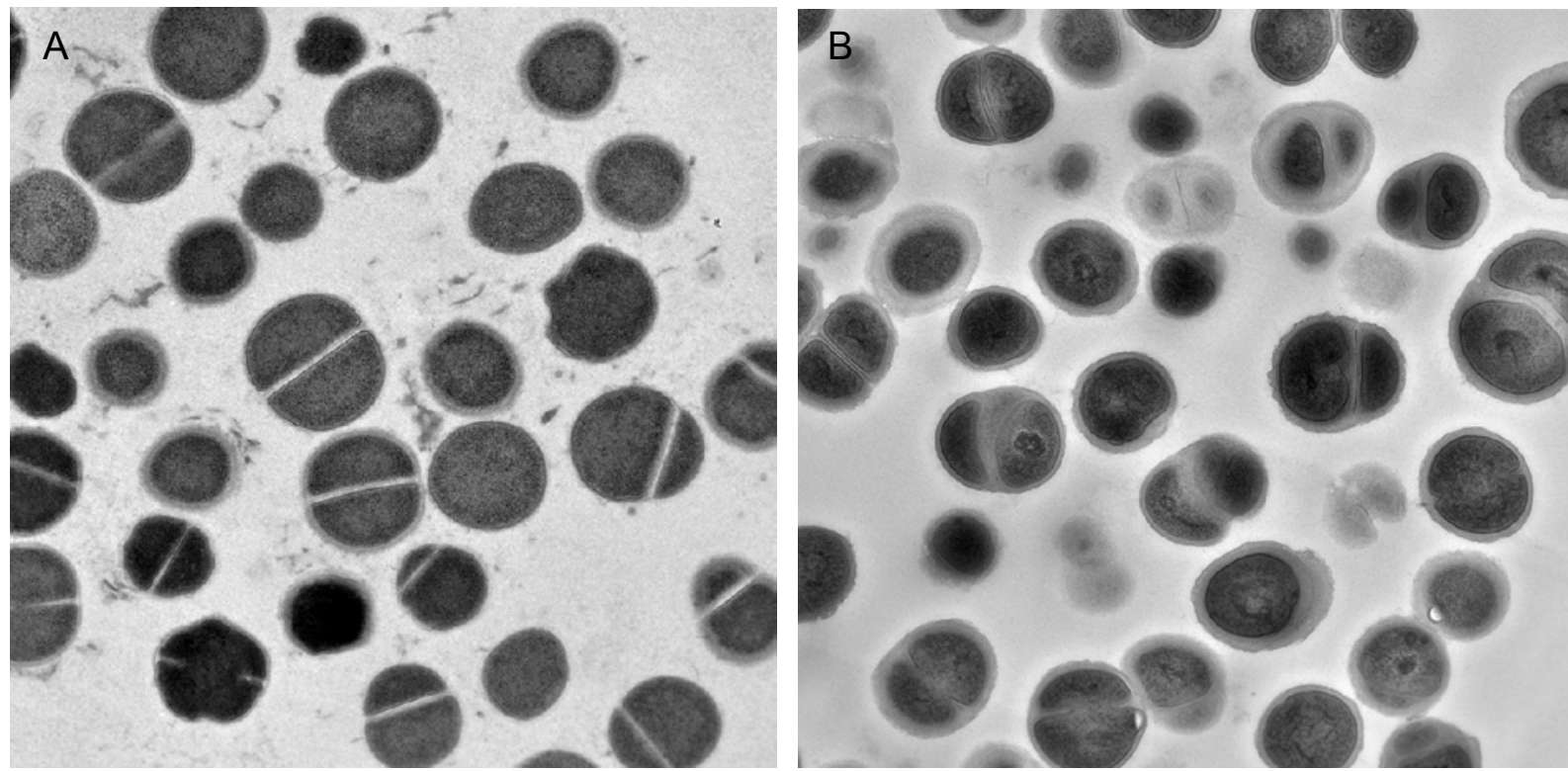

Fig. 1. Cryo-fixed staphylococci of normal colonies are mostly sphere-like, thin-walled cells many of them in division close to completion (A). Cells of SA-SCV (B) comprise a thick wall and irregular cytoplasm, and show disturbance in division.
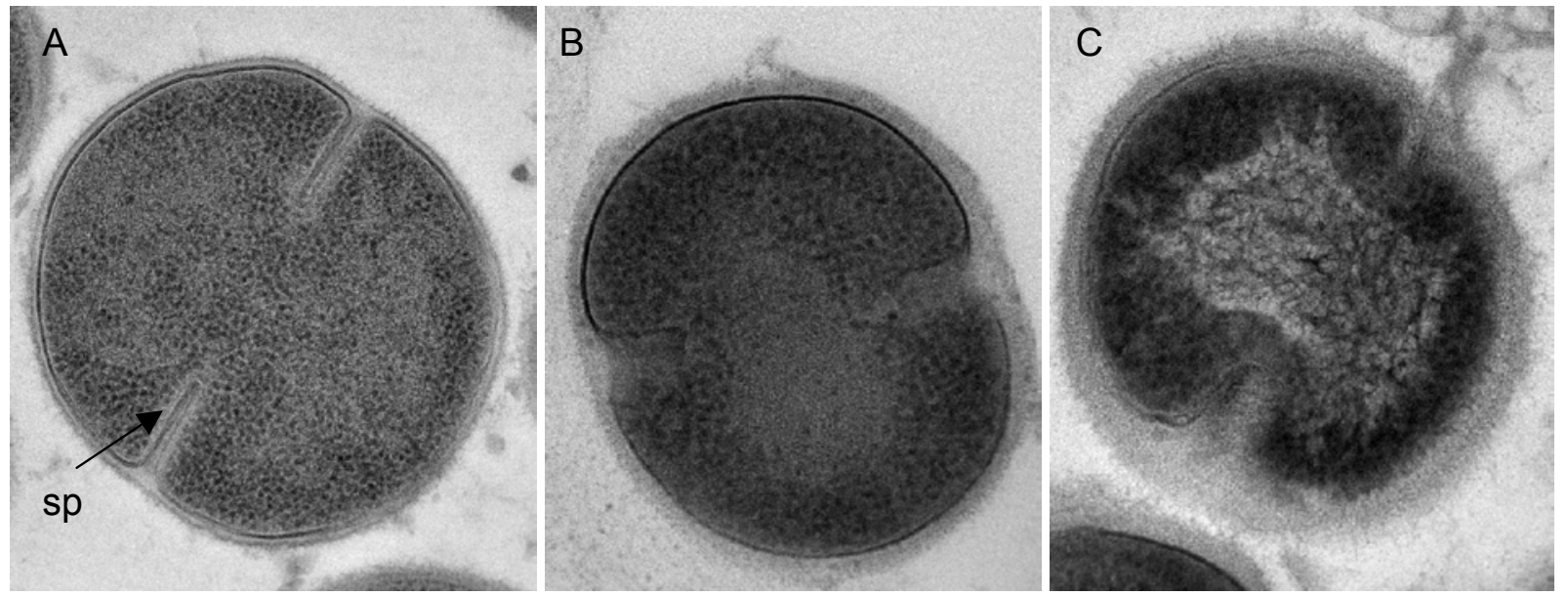

Fig. 2. Cross-section of cryo-fixed staphylococci of normal colonies early in division (A) with thin wall and a distinct splitting system (sp). Note the distribution of the fine granular and rough granular material within the cytoplasm. The splitting system in the thick-walled SA-SCV cells is poorly developed. The rough granular material is condensed at the periphery (B) and becomes confluent (C) while the lucent center is dispersed with fibrillar-like material indicating cell death. 